\title{
The effect of parallax and cadence on asteroid impact probabilities and warning times
}

Peter Vereš ${ }^{1}$ (veres@ifa.hawaii.edu), Davide Farnocchia ${ }^{2}$, Robert Jedicke ${ }^{1}$, Federica Spoto $^{3}$,

Received __ ; accepted _

41 Pages, 11 Figures, N Table

${ }^{1}$ Institute for Astronomy, 2680 Woodlawn Dr, Honolulu, HI, 96822

${ }^{2}$ Jet Propulsion Laboratory, California Institute of Technology, 4800 Oak Grove Drive, Pasadena, CA, 91101

${ }^{3}$ Department of Mathematics, University of Pisa, Largo Bruno Pontecorvo 5, 56127 Pisa, Italy 


\begin{abstract}
We study the time evolution of the impact probability for synthetic but realistic impacting and close approaching asteroids detected in a simulated all-sky survey. We use the impact probability to calculate the impact warning time $\left(t_{\text {warn }}\right)$ as the time interval between when an object reaches a Palermo Scale value of -2 and when it impacts Earth. A simple argument shows that $t_{\text {warn }} \propto D^{x}$ with the exponent in the range $[1.0,1.5]$ and our derived value was $x=1.3 \pm 0.1$. The lowprecision astrometry from the single simulated all-sky survey could require many days or weeks to establish an imminent impact for asteroids $>100 \mathrm{~m}$ diameter that are discovered far from Earth. Most close approaching asteroids are quickly identified as not being impactors but a size-dependent percentage, even for those $>50 \mathrm{~m}$ diameter, have a persistent impact probability of $>10^{-6}$ on the day of closest approach. Thus, a single all-sky survey can be of tremendous value in identifying Earth impacting and close approaching asteroids in advance of their closest approach but it can not solve the problem on its own: high-precision astrometry from other optical or radar systems is necessary to rapidly establish an object as an impactor or close approacher. We show that the parallax afforded by surveying the sky from two sites is only of benefit for a small fraction of the smallest objects detected within a couple days before impact: probably not enough to justify the increased operating costs of a 2-site survey. Finally, the survey cadence within a fixed time span is relatively unimportant to the impact probability calculation. We tested three different reasonable cadences and found that one provided $\sim 10 \times$ higher (better) value for the impact probability on the discovery night for the smallest (10 m diameter) objects but the consequences on the overall impact probability calculation is negligible.
\end{abstract}


Subject headings: Near-Earth Objects; Asteroids, Dynamics 


\section{Introduction}

The past quarter century has witnessed an exponential increase in the number of known near-Earth objects $\mathrm{I}^{1}$ (NEO) accompanied by a concomitant improvement in the ability to calculate their impact probabilities with Earth. The job of identifying the largest and most hazardous NEOs, those larger than about $1 \mathrm{~km}$ diameter, has mostly been accomplished and asteroid surveys are now focussing on the individually less hazardous but far more numerous smaller asteroids. The large asteroids can be detected at great distances years to centuries in advance of their impact but the smaller asteroids may only be detected on their final approach, if at all, since about $40 \%$ of them must approach from the direction of the Sun in daylight sky. This work quantifies how the impact probability and warning time evolve in the impact apparition for the smaller asteroids as a function of their size, time after discovery, and observing cadence. In particular, we examine whether the parallax afforded by observations at nearly the same time from two independent observatories provides leverage in improving the impact probability calculation or increasing the impact warning time.

The Catalina Sky Survey (CSS, Larson et al. 1998) and Pan-STARRS 1 system (Pan-STARRS; e.g. Kaiser et al. 2002; Hodapp et al. 2004) currently dominate the field of NEO discovery — almost $90 \%$ of all NEOs and about $75 \%$ of all potentially hazardous object: $^{2}$ (PHO) were discovered by these two surveys in calendar years 2012 and 2013. The known population of NEOs larger than $1 \mathrm{~km}$ diameter is $>90 \%$ complete (Mainzer et al. 2011) so the discovery rate of NEOs in this size range has decreased by about a factor of 6 from a peak of 93 in the year 2000 to about 15/year in the last two years.

\footnotetext{
${ }^{1}$ NEOs are asteroids or comets that have a perihelion distance $<1.3$ au

${ }^{2}$ PHOs are NEOs that have a minimum orbital intersection distance (e.g. Gronchi 2005) with Earth of $<0.05$ au and absolute magnitude $H<22$
} 
Despite the success of the surveys in the past few decades it remains the case that the most likely warning time for an impact is zero - contemporary surveys are unlikely to detect smaller but still dangerous asteroids because they do not survey the entire sky deeply or regularly enough to identify the next impactor. The surveys are further limited by the simple fact that ground-based facilities can not survey during the day and about $40 \%$ of all impactors will approach from the direction of the Sun. These problems were spectacularly highlighted by the Chelyabinsk impact on the morning of 15 February 2013 (e.g. Brown et al. 2013; Borovička et al. 2013) — with absolutely no warning a $\sim 17 \mathrm{~m}$ diameter object blew up in the atmosphere with an energy equivalent to about 500 kilotons of TNT, damaging buildings $50 \mathrm{~km}$ away in the city of Chelyabinsk and injuring about 1,500 people.

The impact risk associated with the unknown objects $>1 \mathrm{~km}$ diameter is now comparable to the impact risk with the much more numerous but individually less destructive objects with diameters $<1 \mathrm{~km}$. The new balance in the impact risk, along with the realization that smaller impacts may be more numerous but less destructive than anticipated a decade ago (e.g. Brown et al. 2013), has contributed to an increased interest and funding for the NEO survey programs in recent years. e.g. NASA's NEO Observations (NEOO) program office now solicit: $3^{3}$ proposals for surveys that "provide capability to detect the subset of $90 \%$ of PHOs down to 140 meters in size'

The smaller NEOs are more difficult to detect than the larger ones, will be detected (if they are detected) closer to Earth, and consequently have shorter observational arcs. The limited time range of the set of detections can make it difficult to identify real impactors even during the apparition in which the impact will take place. This was not the case for

\footnotetext{
${ }^{3}$ ROSES 2011 NEOO solicitation section C.9.1.1.
} 
the few-meter diameter asteroids $2008 \mathrm{TC}_{3}$ (e.g. Jenniskens et al. 2009) and $2014 \mathrm{AA} 4$, the only natural objects to be discovered before striking Earth. The very smallest objects will be discovered so close to Earth that, if individual detections of the object can be associated with one another as a 'tracklet' (Denneau et al. 2013), the non-linear motion of the detections on the sky-plane due to topocentric parallax can provide enough leverage in the orbit solution to predict an impact.

The observable characteristics of NEOs that will impact Earth can be quite different from those of other NEOs (e.g. Farnocchia et al. 2012; Vereš et al. 2009; Chesley and Spahr 2004). For instance, their observable steady-state distribution on the sky-plane is a function of their size and time before impact. Decades before impact they tend to be concentrated in 'sweet spots' near the ecliptic and within about $120^{\circ}$ of the Sun. As the time until impact decreases from weeks to days they spread out over most of the sky but there are still concentrations in the direction looking towards and away from the Sun. An object on its 'death plunge' must be moving directly towards Earth in a geocentric reference frame so that about a week before impact its apparent rate of motion may be small — likely mimicking the rate of motion of much more distant and totally harmless asteroids and perhaps not triggering followup that would allow an impact probability calculation.

The techniques employed for the impact probability calculation have evolved dramatically over the past few decades with the realization that asteroid impacts have shaped the Moon's surface and influenced the evolution of life on Earth. Indeed, it was only 34 years ago that Alvarez et al. (1980) proposed that the KT extinction was the result of an asteroid impact and, even though Opik (1952) stated that "Over a dozen meteor craters are at present known on the earth's surface", it was only in 1960 that Chao et al. (1960) found strong physical evidence that Meteor Crater in Arizona, USA, was formed in

\footnotetext{
${ }^{4}$ Minor Planet Electronic Circular 2014-A02
} 
an impact event.

Opik (1952)'s estimated collision rates using the 'Theory of Probabilities' for the entire NEO population were surprisingly good given that only six NEOs were known at the time. His collision probability formulae formed the basis of much of the impact collision work in the next decades (e.g. Bottke et al. 1996; Kessler 1981) but were eventually supplanted by new numerical techniques (Milani et al. 2002). The two primary operational asteroid impact warning systems, the Jet Propulsion Laboratory's Sentry system and the NEODyS CLOMON2 system, calculate the collision probability by generating synthetic 'Virtual Asteroids' (VA) on orbits that are consistent with the known set of observations and propagating all of them into the future with a N-body integrator to search for impacts (Milani et al. 2005a). These impact warning systems are based on a geometric sampling technique for which the identification of the Virtual Impactors (VI) is performed on the line-of-variation (LOV, Milani et al. 2005b) thus avoiding the poor efficiency inherent to the Monte Carlo methods, especially when the collision probability is small.

Impact predictions are extremely sensistive to the orbit accuracy which depends on many factors but the primary drivers are the length of the observational arc and the astrometric accuracy (e.g. Desmars et al. 2013). The longer the arc and the better the astrometry the more accurate the orbit. The latter effect is best illustrated by radar detection of asteroids that provide exquisite range and range-rate information thereby dramatically improving the impact probability accuracy and/or extending the time frame during which the impact probability can be calculated (Ostro et al. 2002).

Impactors can be either direct or resonant (Milani et al. 1999). Direct impactors collide with Earth during their first known encounter and must be discovered far away to have a large warning time. The warning time for small impactors can be significantly less than one orbital period because they have to be close to Earth to be detected. On the other 
hand, resonant impactors experience intervening Earth encounters before collision. The intervening encounters are the main source of non-linearity in the dynamics and usually prevent a conclusive assessment of the impact threat but provide additional observational opportunities to detect and constrain their orbits and the impact threat.

In this work we focus on the evolution of the collision probability with time for a single survey and concentrate on direct Earth impactors that are detected in the apparition during which the impact occurs. The smaller the asteroid the more likely this scenario as the likelihood that small asteroids will be detected in earlier apparitions is 1) small and, even if they are detected, 2) it is unlikely that they will be recoverable in future apparitions because of the large uncertainties in their ephemeris based on the short observational arcs in the discovery apparition. Thus, we concentrate on collision probability evolution with time for $300 \mathrm{~m}, 100 \mathrm{~m}, 50 \mathrm{~m}$ and $10 \mathrm{~m}$ diameter impactors.

We also explore whether the collision probability calculation benefits from simultaneous or nearly-simultaneous parallax measurements from two observatories. The heliocentric motion of the impactor and Earth as well as the topocentric rotation of the observer about the geocenter produce 'parallax' between successive observations of the same object even from the same site. For very close objects that will impact within days of discovery there may be benefits from the two-site scenario - especially in rapidly identifying the object as an impactor.

Finally, we measure the single-system impact warning time as a function of impactor diameter. We expect the warning time to be longer for larger objects but the exact relationship between diameter and warning time is not intuitively obvious. The larger objects are discovered at greater distances where their rate of motion is similar to the much more distant main belt objects and the impact probability will be much smaller. If the impact probability is too small it may not cross the threshold to flag the object as an 
imminent impactor.

\section{Method}

\subsection{Synthetic asteroid populations}

Our study considers three different classes of asteroids that might be identified soon after discovery as impactors:

\section{- Impactors}

We used a 133 member subset of the population of Earth impactors developed for Vereš et al. (2009) that strike the Earth in a 12 month period beginning at the same time as the 12 month survey simulation described below $(2.2)$. The impactors' orbit

elements are drawn from a realistic population of NEOs (Bottke et al. 2002) and tend to have perihelia or aphelia that lie near Earth's geocentric distance of about 1 au (fig. 11). These types of orbits are nearly tangent to Earth's orbit so they spend more time available for impact. There is also an enhancement with small inclinations for the same reason.

- Close Approachers

We generated 8,275 synthetic asteroids from the Bottke et al. (2002) NEO model that approach Earth to within $10 \mathrm{LD}$ (lunar distances, $\lesssim 0.028 \mathrm{au}$ ) during the one year simulated survey $(\$ 2.2)$. The orbit distribution of the close approachers is more representative of the underlying NEO population (fig. 1) and has a higher mean eccentricity and inclination compared to the impactors. This leads to them having higher speeds relative to Earth and higher apparent rates of motion as viewed from ground-based observatories (compared to the impactors). The number of close approachers increases linearly with the impact parameter because the area of annuli 
of fixed width increases linearly with the annuli diameter (fig. 22).

\section{- Main Belt Objects (MBO)}

There are about one million asteroids larger than $1 \mathrm{~km}$ diameter in the main belt with semi-major axes between about $2.0 \mathrm{au}$ and $3.5 \mathrm{au}$ (fig. 1). By definition, they can not approach within $\sim 0.7$ au of Earth but tens of thousands will be within the detection limits of the survey that we model below $(\$ 2.2)$ and many of them will not be known objects (at least in the beginning of the survey). We will show that the objects' rates of motion are typical of some incoming impactors and we wanted to determine if a relatively large astrometric uncertainty could generate false non-zero impact probabilities even for these distant objects. We used a sample of about 14,000 synthetic main belt asteroids selected from the Grav et al. (2011) solar system model that have minimum perihelion magnitudes $5^{5}$ detectable in our synthetic survey $(V<20)$. We used the absolute magnitudes $(H)$ from the Grav et al. (2011) model that were assigned randomly according to a realistic size-frequency distribution.

\subsection{Survey simulation}

We simulated the detection of small incoming impacting asteroids with the indevelopment ATLAS (Tonry 2011) system because of its all-sky every-night survey capabilities. The smaller the asteroid the more likely it is that it will not be brighter than any of the contemporary or planned survey system's limiting magnitudes until a few days before impact, so that detecting the object requires nearly all-sky coverage over that time interval. ATLAS achieves all-sky coverage by using small telescopes with wide fields-of-view

\footnotetext{
${ }^{5}$ The minimum perihelion magnitude is the apparent magnitude an object would have if observed at opposition from Earth when the object is at perihelion.
} 
(FOV) and large-format CCD cameras. Thus, it has a relatively large pixel scale (and astrometric uncertainty) and brighter limiting magnitude than other surveys.

The two primary purposes of this study were to 1) measure the time-evolution of the impact probability of asteroids detected with a realistic survey and 2) measure the effect of parallax on the impact probability precision.

To address the first issue we generated synthetic observations of each of our synthetic asteroids for a simulated one year ATLAS survey. Our low-fidelity instantiation of the survey covered the entire dark sky each night without regard for the Moon, weather, galaxy and clouds. The survey does account for the changing duration of the night through the year and geometrical constraints from the horizon. The fidelity of the simulation is not critical to the two primary purposes of this study but will have an impact on e.g. the calculated detection efficiency for small objects.

We addressed the second issue by using the synthetic survey to simulate the performance of two ATLAS surveys located at observatory sites F51 and 568 (respectively, the locations of the Pan-STARRS 1 facility on Haleakala, Maui, Hawaii, and the University of Hawaii $2.2 \mathrm{~m}$ telescope on Mauna Kea, Hawaii). These locations are amongst the best ground-based astronomical sites in the world with typically $>75 \%$ clear nights, dark sky, high altitude, sub-arc-second seeing, and a number of other operational observatories and instruments. The sites are separated by $\sim 130 \mathrm{~km}$ to enable parallax measurements for nearby asteroids. For instance, an asteroid at $10 \mathrm{LD}$ can have a parallax of up to $\sim 6.5^{\prime \prime}$ from the two sites — about an order of magnitude larger than the system's astrometric uncertainty. Increasing the distance between the two observatories would enhance the parallax effect. However, the meteorological correlation would be lower and it would be less likely that both sites could observe. Furthermore, the greater the separation between the two sites the more difficult it is to survey the same fields. Finally, two separate sites 
introduces additional cost and management issues.

To create the survey we divided the sky into 'square' tiles (or fields) with each square having an area equal to that of an ATLAS camera's FOV of $\sim 40 \mathrm{deg}^{2}$. The tile centers were equally spaced at about the width of the ATLAS FOV along lines of latitude. They were also spaced in latitude by the width of the ATLAS FOV. This pattern is not optimal because it results in significant field overlap at high latitudes but it was simple to implement and the details of the survey pattern will have little impact on the results. We used the Tools for Automated Observing optimization (TAO) packag $\complement^{7}$ to schedule the nightly surveying of the tiles and maximize the number of fields exposed each night with the desired cadence subject to the survey's limitations. Each tile was visited 4 times per night with roughly a Transient Time Interval (TTI) of 15 minutes between visits. We did not account for the Moon, galactic plane, planets, bright stars or weather (fig. 3) but did account for the camera readout and telescope slew times. The fields were observed only when the Sun was more than $12^{\circ}$ below the horizon and the field centers were more than $30^{\circ}$ above the horizon (i.e. above 2 airmasses). We imposed a southern declination limit of $-30^{\circ}$ that will have the effect of decreasing the detection efficiency for the imminent impactors that are concentrated towards opposition, but will provide more time for surveying the 'sweet spots' (Chesley and Spahr 2004) at small solar elongations where the sky-plane density of future and larger impactors is highest. The strategy of surveying the sweet spots is a likely scenario for actual surveys that are rightfully more concerned with the long-term advance notice of larger impactors rather than the short-term notice for smaller objects.

We used the Pan-STARRS 1 Moving Object Processing System (MOPS, Denneau

${ }^{6}$ Some of the values for the ATLAS system characteristics used in this work represent early expectations for the system. The exact values have no impact on our general conclusions.

${ }^{7}$ Paolo Holvorcem, http://sites.mpc.com.br/holvorcem/tao/readme.html 
et al. 2013) to generate the synthetic asteroid detections in our simulated survey and to link detections within a tile on one night into 'tracklets'. MOPS employes an N-body integrator and the DE406 ephemerides (Standish 1998) to compute the position and brightness of every synthetic detection. In practice, we set the absolute magnitude of each synthetic object to $H=0$ and turned off the MOPS system's capability of adding astrometric and photometric uncertainty to each detection so that we could modify those values post hoc as described below.

\subsection{Photometric \& astrometric uncertainty}

Each synthetic MOPS detection had a calculated apparent magnitude $m_{0}$ corresponding to the value if the object's absolute magnitude $(H)$ was zero. We could then assign any other absolute magnitude to the object and its actual apparent magnitude corresponding to that detection would than be $m^{*}=m_{0}+H$. We then used a reported apparent magnitude $m=m_{0}+H+\Delta m$ where $\Delta m=\max \left\{0.01, G\left[0, \sigma\left(m^{*}\right)\right]\right\}$ and $G$ represents a randomly generated number from a normal (Gaussian) distribution with a mean of zero and width $\sigma\left(m^{*}\right)=0.02 \times 2^{\left(m^{*}-16\right)}$ appropriate for the ATLAS system. The max function limits the minimum photometric uncertainty to $0.01 \mathrm{mag}$.

We did not account for the effect of trailing of the detections due to the motion of the object during an exposure (e.g. Vereš et al. 2012). The neglect is justified because the large ATLAS plate scale of about $2^{\prime \prime} /$ pixel means that detections trail by $<1$ pixel for rates of up to $1.6^{\circ}$ day and the majority of the impactors that are $>10 \mathrm{~m}$ diameter move slower than this rate when they are first detected (fig. 5). Main belt objects are not trailed at all because they typically move fastest near opposition at rates of about $0.25^{\circ} /$ day. The close approachers can move much faster but here we assume that ATLAS will apply a trail finding and fitting algorithm to maintain astrometric and photometric integrity for objects 
with faster rates motion.

The impact probability calculation depends on the reported astrometric uncertainty that, in turn, depends on the apparent brightness of the detections and the system's pixel scale. The reported astrometric position of each synthetic detection was 'fuzzed' by an offset $\Delta=\max \left\{0.1^{\prime \prime}, G\left[0, \sigma_{p}\left(m^{*}\right)\right]\right\}$ where the sub-script 'p' represents 'positional' uncertainty and $\sigma_{p}\left(m^{*}\right)=2^{\prime \prime} / 10^{8.5-0.4 m^{*}}$ appropriate to the ATLAS system's $2^{\prime \prime}$ pixel scale and photometric performance. The max function ensures that the minimum astrometric uncertainty is always $>0.1^{\prime \prime}$.

\subsection{Survey cadence}

One of the main goals of this study was to demonstrate how two survey sites could improve the impact probability determination in comparison with a single site, but there are many different survey cadences that could be implemented in either scenario. We decided that a fair comparison between the two scenarios required maintaining the same combined tracklet arc-length (i.e. time from first to last observation) and studied three different 2-site visit cadences (see fig. 3):

- 1-site (quads)

4 images acquired with roughly a TTI between each

- 2-site no-shift

2 images acquired at each site with roughly 3 TTI between them

- 2-site half-shift

2 images acquired at each site with roughly 2 TTI between them interleaved with the other site 
- 2-site full-shift

2 images acquired at each site with roughly a TTI between them sequential with the other site

We will show in $\$ 3.3$ that there is little difference in performance between the cadences but there is a marginal benefit to the 2-site full-shift scenario and we adopt it as the nominal cadence unless otherwise specified.

\subsection{Observability windows}

For a fair comparison between the the observation circumstances of our synthetic close approachers and impactors we only considered observations of close approachers before the moment of closest approach. This requirement is symmetric with the impactors because it is impossible to obtain observations of an impactor after impact.

Furthermore, because our simulated survey was only one year in duration instead of infinite, we were careful to consider only those impactors of different sizes that could be detected before impact. To do so we defined an impactor diameter $(D)$ dependent 'time observability window', $t_{\text {window }}(D)$, and then, letting $t_{\text {begin }}$ and $t_{\text {end }}$ represent the simulated survey's starting and finishing time respectively, require that the time of impact of an object with diameter $D, t_{\text {impact }}(D)$, satisfy $t_{\text {impact }}(D) \geq t_{\text {begin }}+t_{\text {window }}(D)$ and $t_{\text {impact }}(D) \leq t_{\text {end }}$. i.e. we require that the time of impact and the entire observability window be in the simulated survey time.

We defined $t_{\text {window }}(D)$ using our simulated survey and synthetic objects from which we could measure the number of days between the first detection and impact as a function of the impactor diameter (fig. 4). If $\bar{t}_{\text {first }}(D)$ represents the average value as a function of diameter and $\sigma_{\text {first }}(D)$ the standard deviation of the distribution then we set 
$t_{\text {window }}(D)=\bar{t}_{\text {first }}(D)+\sigma_{\text {first }}(D)$. This time observability window encompasses the actual observability window of about $84 \%$ of the objects at each diameter but will eliminate the $\sim 16 \%$ of the sample with the longest observability times. The choice reflects a balance between increasing the time observability windows and keeping more objects in the analysis - longer windows mean fewer objects satisfy the requirement. No window was applied for main belt asteroids.

\subsection{Orbit determination and impact probability}

Orbit determination is the process of identifying the best-fit least-squares orbit to the astrometric dataset. We used the standard differential correction procedure (Milani and Gronchi 2010, Chap. 5) with the objects's synthetically generated orbit as the starting point. The use of the synthetic orbit as the initial orbit in the fit will skew our orbit determination and impact probability calculation towards more accurate values than could be expected from the single-survey performance in our study. We do not consider this an important issue because the NEO candidate followup community rapidly provides additional astrometry for initial orbit determination by the Minor Planet Center. It is also worth noting that we assume a high efficiency and accuracy for linking detections of the same object into tracklets and then linking the tracklets into 'tracks' Milani and Gronchi 2010, Chap. 8) but this is justified because the MOPS has $>99.5 \%$ efficiency at doing so (Denneau et al. 2013).

After an orbit was computed along with its corresponding uncertainty we computed the probability of an Earth impact. The analysis of close encounters is typically a strongly non-linear problem whose solution requires sophisticated methods (e.g., Milani et al. 2005a) but we adopted a simplified approach to the calculation of the impact probability since this study only assesses its evolution in the days and weeks before impact. First we search for 
upcoming close approaches and then we perform a linear mapping of the orbital uncertainty region to the close encounter $b$-plane and compute the impact probability corresponding to the intersection between the mapped uncertainty region and Earth's cross section (Valsecchi et al. 2003). The short-term propagation and the lack of intervening planetary encounters justifies the adoption of the simplified linear approach.

We updated the orbit determination and risk assessment night-by-night within the simulation to assess the time evolution of the impact probability. i.e. we incrementally added each tracklet to an object's astrometric data set and then recalculated the orbit and associated impact probability each night.

\section{Results \& Discussion}

We tested the performance of the ATLAS survey for impactors and close approachers of $5,10,20,50,100,150$, and 300 meters diameter because we expected there to be a size-dependency on the ability to rapidly calculate impact probabilities. Large objects will typically be detected at larger distances where the effects of parallax are small and the lever arm to impact is large, making it difficult to assign a high probability to a possible impact. Small objects will be detected close to Earth so parallax will provide some power in the orbit solution and impact probability calculation.

\subsection{Observable characteristics of the impactor population}

The mean apparent rate of motion of impactors on the first night of detection is about $0.25 \pm 0.05 \mathrm{deg} /$ day, essentially matching both the mean rate and distribution of typical main belt asteroids moving at $0.19 \pm 0.06 \mathrm{deg} /$ day (fig. 5). The fastest impactor rates of $\gtrsim 0.5 \mathrm{deg} /$ day mimic that of the perfectly harmless Hungaria asteroids on the inner edge 
of the main belt (e.g. Jedicke 1996; Rabinowitz 1991) that can also have very high rates of motion in ecliptic latitude because of their high inclinations. On the other hand, the Earth-grazing close approachers have high apparent fly-by speeds even on their night of first detection ranging up to $10 \mathrm{deg} / \mathrm{day}$ and $<2 \%$ have rates of $<0.5 \mathrm{deg} /$ day in the range of the typical impactor.

Figure 6 illustrates several features of the detected impactors's sky plane motion. First, smaller objects are visible for much less time than the larger impactors, the impactors typically move westwards and mostly in longitude, and they are first detected near the system's limiting magnitude and increase in brightness as they move closer to Earth. The larger objects can be discovered almost everywhere on the night sky but the smaller objects tend to be discovered towards opposition where the reduction in apparent brightness due to phase angle effects is smallest.

\subsection{Detection efficiency \& rates}

The ATLAS pre-impact detection efficiency (figs. 7) plateaus at a maximum of about $50 \%$ even for the largest objects for geometrical reasons - ATLAS only surveys about half the sky but detects everything brighter than its limiting magnitude. The efficiency decreases for smaller objects with a particularly dramatic drop from $10 \mathrm{~m}$ to $5 \mathrm{~m}$. i.e. it decreases by about half, to about $25 \%$, from $300 \mathrm{~m}$ to $10 \mathrm{~m}$ diameter — nearly 3 orders of magnitude in the impactors cross-sectional area — but then decreases by $>50 \%$ from $10 \mathrm{~m}$ to $5 \mathrm{~m}$ diameter (only a factor of 4 in cross-section). In this size range the objects are typically well below the survey system's limiting magnitude and even a nightly cadence is not sufficient to catch the objects in the brief time they are bright enough to be detected before impact. 
The behavior of the detection efficiency as a function of diameter is different for close approaching asteroids (figs. 7). Remembering that for a direct comparison to the impactors we only considered close approachers detected before closest approach, the detection efficiencies are identical at the largest sizes we considered. The close approacher detection efficiency would have been much higher had we allowed them to be detected after close approach because there would be much more time to detect them and because objects that approach in daylight from the direction of the Sun are likely to depart and be detectable in the night time sky. The detection efficiency is slightly higher for the close approachers in the $50 \mathrm{~m}$ to $150 \mathrm{~m}$ diameter range because these objects will be bright enough and detectable longer than the impactors. On the other hand, close approachers of $<50 \mathrm{~m}$ diameter are detected less efficiently than the impactors because they are usually too far away and therefore too faint to be detected - $1 \%$ of the close approachers in our simulation come no closer than $1 \mathrm{LD}$ while all the impactors do so by definition.

Figure 7 illustrates that it is unlikely that ATLAS will detect impactors larger than $5 \mathrm{~m}$ diameter. There are simply not enough of them and the detection efficiency for the smaller, most frequent, impactors is $<10 \%$. It is dangerous to extrapolate ATLAS's ability to detect even smaller impactors in the $1-2 \mathrm{~m}$ diameter size range like $2008 \mathrm{TC}_{3}$ (e.g. Jenniskens et al. 2009) because the detection efficiency becomes particularly sensitive to the observing cadence and subtleties of image processing e.g. trail detection. However, assuming that the detection efficiency drops to just $1 \%$ for a $1 \mathrm{~m}$ diameter object ATLAS might detect one impactor every few years.

On the other hand, ATLAS will detect 100s or 1,000s of close approaching asteroids to within $10 \mathrm{LD}$ because there are far more of them than impactors (scaling like the ratio of the cross sectional area of a circle with radius equal to the close approach distance and the cross sectional area of Earth). Most of the detected close approachers will be in the 
$20 \mathrm{~m}$ to $50 \mathrm{~m}$ diameter range (fig. 7) but there will still be $10 \mathrm{~s}$ to 100 s of detected objects of $<20 \mathrm{~m}$ and $>50 \mathrm{~m}$ diameter. We will show in $\$ 3.4$ that ATLAS alone can not establish that all the close approachers are not impactors - 100s of the closest approaching objects will always have a residual non-zero impact probability unless additional observations are acquired with other optical or radar facilities.

\subsection{Impact probability evolution for impacting asteroids}

The impact probability for an impacting asteroid depends on the observed arc length and the object's distance from Earth and both depend on the size of the impactor (see fig. 8). Longer arc lengths provide better orbit precision and a higher impact probability because all these objects are actually impactors. However, when an object is far from Earth the integration to the impact epoch stretches the orbital uncertainty and decreases the impact probability. The impact probability is smaller for larger diameter impactors at the same number of nights after discovery because smaller objects are likely to be observed when close to Earth and therefore closer to their impact time. The topocentric parallax in the detections and deviation from great-circle motion allow a better orbit determination and the small amount of time to impact provides a higher impact probability.

The typically monotonically increasing impact probability that asymptotically

approaches unity (fig. 8) is the expected behavior though we were surprised that the singlesystem astrometry required about a week to reach $\sim 100 \%$ for the $50 \mathrm{~m}$ diameter impactors and a month to reach the same values for the $100 \mathrm{~m}$ diameter impactors. The impact probability reached $99 \%$ an average of $2.0 \pm 0.5,4.5 \pm 1.8,8.9 \pm 4.7$ and $28.7 \pm 16.3$ days before impact for impactors of $10 \mathrm{~m}, 50 \mathrm{~m}, 100 \mathrm{~m}$, and $300 \mathrm{~m}$ diameter at mean geocentric distances of $0.6 \pm 0.4 L D, 1.0 \pm 0.5 L D, 1.9 \pm 1.0 L D$ and $5.5 \pm 1.5 L D$ respectively. Thus, even though a $300 \mathrm{~m}$ diameter object has nearly $1000 \times$ the cross-sectional area of 
a $10 \mathrm{~m}$ diameter object, and is therefore about a $1000 \times$ brighter $(\sim 7.5 \mathrm{mag})$ at the same topocentric distance, an imminent impact becomes definitive with only about $10 \times$ more warning time when the larger object is only $\sim 5 \times$ further away.

Of course, our calculated impact probabilities rely only on the detections from a single survey with relatively large astrometric uncertainty compared to contemporary standards (because of its all-sky coverage as described in \$2.3). In some cases there will be nights with particularly bad astrometric error that temporarily decrease the impact probability as illustrated by the $100 \mathrm{~m}$ diameter example in fig. 8. The detection of a real impactor with even a $10^{-6}$ impact probability would certainly trigger high-precision ground-based optical and radar followup that would improve the orbit determination and impact probability.

None of the four objects represented in fig. 8 had an impact probability calculated on the first night. This does not mean that the impact probability was zero, only that with just one night's data the orbit determination could not converge (even with our method where we start with the correct initial orbit) on a full 6-parameter orbit along with its covariance matrix and both are required for the impact probability calculation. While short-arc orbit determination methods are available they have not yet been extensively tested in their ability to provide reliable hazard assessments (e.g. Virtanen et al. 2001; Milani and Knežević 2005; Chesley 2005). With our single survey simulation there is a $\sim 14 \%$ efficiency for calculating an impact probability on the first night for $10 \mathrm{~m}$ diameter objects with the efficiency decreasing with diameter to just $2 \%$ for $300 \mathrm{~m}$ impactors. Therefore, it is critical to have rapid follow-up observations from other observatories to achieve a better efficiency in recognizing impactors on the discovery night — but there may be nothing particularly noteworthy about the tracklet to flag it as worthy of immediate follow-up.

The average impact probability as a function of time for an ensemble of impactors of a specific diameter (fig. 9 behaves much the same as the individual examples illustrated in 
fig. 8. The four survey cadences $(\$ 2.4)$ are essentially equivalent in terms of the numerical value and efficiency of the impact probability calculation beginning on the second night after discovery. While the efficiency for calculating an impact probability on the first night is small (discussed above), the 2-site full-shift scenario is always superior, so that combining observations from different stations does provide better constraints on the orbit and allows for an impact probability calculation. Again, we stress that this is true only on the first night of discovery and only for the small fraction of objects for which an impact probability can be calculated, so the benefits of parallax in the impact probability calculation afforded by a 2-site scenario is limited to a small fraction of the least dangerous impactors. We recognize that the 2 -site scenario offers other advantages such as increased immunity to weather shutdowns (one site can still survey if the other is in-operational) and natural disasters (such as lava flows) but at the expense of having to maintain two sites.

We define the impact warning time as the time interval between the impact epoch and the epoch at which an object's Palermo Scale (Chesley et al. 2002) ranking becomes $>-2$. We used the Palermo Scale because it is a standard tool to communicate the risk posed by a possible impact and selected the -2 threshold as it corresponds to cases that 'merit careful monitoring' 8 . The warning time increases with the impactor diameter as $t_{\text {warn }} \propto D^{1.1 \pm 0.2}$ (fig. 10 - detected impactors with an orbit determination that are smaller than $\sim 20 \mathrm{~m}$ diameter typically have less than a couple of days of warning time, $50 \mathrm{~m}$ diameter objects provide about one week notice, and the warning time is weeks to months for objects $>100 \mathrm{~m}$ diameter. Including those objects that were detected but for which an orbit was impossible to calculate (even starting with the correct orbit as the initial value in the fitting procedure) the warning time increases with diameter as $t_{\text {warn }} \propto D^{1.1 \pm 0.2}$.

The time to impact from first detection $(\Delta t)$ should increase linearly with diameter

\footnotetext{
${ }^{8}$ http://neo.jpl.nasa.gov/risk/doc/palermo.html
} 
$D$ because the geocentric distance $\left(\rho_{\text {limit }}\right)$ at which an asteroid becomes brighter than a system's limiting magnitude $\left(m_{\text {limit }}\right)$ is given by

$$
5 \log _{10} \rho_{\text {limit }}=m_{\text {limit }}-H+\phi\left(r, \rho_{\text {limit }}\right)
$$

where $\phi$ is a 'phase function' that depends on the objects geocentric and heliocentric $(r)$ distance (Bowell et al. 1988). Furthermore, $H \propto \log _{10} D$ (Pravec and Harris 2007) so it follows that $\rho_{\text {limit }} \propto D$ assuming that $\phi$ is roughly constant (which is justified given that the heliocentric distance is nearly constant during the final approach $(r \sim 1)$ and because $\phi$ depends on the phase angle, which does not change much during an impacting object's

final approach to Earth). Since $\rho_{\text {limit }}=v \Delta t$ where $v$ is the speed of the impactor relative to Earth it follows that $\Delta t \propto D$.

However, $v$ depends on the diameter because smaller objects are detected closer to Earth where they are moving faster because they have accelerated in Earth's gravity well. Assuming that impactors fall towards Earth with similar initial $v_{\infty}$ then it is not difficult to show that the speed at discovery goes roughly as $v \propto D^{1 / 2}$ and that $\Delta t \propto D^{3 / 2}$.

The impact warning time $t_{\text {warn }}$ as we have defined it is related, but not identical to, and always $\leq \Delta t$. Thus, we expect that $t_{\text {warn }} \propto D^{x}$ with $1<x<1.5$ in agreement with our measured value of $1.3 \pm 0.1$. To put this in perspective, a Chelyabinsk-like impactor of $20 \mathrm{~m}$ diameter would typically only have a 2 day warning time if it was detected by an ATLAS-like survey.

\subsection{Impact probability evolution for main belt and close approaching asteroids}

We included a sample of main belt and close approaching asteroids to assess the survey's ability to distinguish them from impactors. The problem may be difficult when 
only a short arc of observations is available in which case the orbital uncertainty is large and there may be multiple and very different initial orbits (Milani et al. 2008) consistent the observations, some of which lead to a least-square solution far from the actual orbit.

The inclusion of the distant, slow-moving main belt asteroids may seem surprising but fig. 5 illustrates that the rate of motion of impacting asteroids can be similar to that of main belt objects. Despite the similarity in their rates of motion we never found a non-zero impact probability for a synthetic main belt object with $\geq 2$ days of arc. It is worth noting once again that we used the actual main belt orbit as the starting point to obtain the orbit solution — this process biases our results towards decreasing the calculated impact probability a short time after discovery.

The most likely false impactors must be PHOs that experience a close Earth approach. These objects might be identified as they approach Earth and the astrometric uncertainties and orbit integration may combine to produce non-zero impact probabilities. Indeed, about $30 \%$ of the close approaching objects in our synthetic population had an impact probability $>10^{-6}$ at some time during the simulation $9^{9}$ but none of the impact probabilities ever exceeded $3 \%$.

With our single-system survey simulation (i.e. one or two sites) we find that even large objects, those $\geq 50 \mathrm{~m}$ diameter, can have non-zero impact probabilities just a few days before impact (fig. 11). About 3 to $5 \%$ of the $50 \mathrm{~m}$ and $100 \mathrm{~m}$ diameter close approachers have a persistent impact risk on the day of (false) impact which means that follow-up observations from other stations are critical to establish the lack of danger from these objects. A persistent impact risk remains on the day of impact for about $75 \%$ of the $10 \mathrm{~m}$

\footnotetext{
${ }^{9} 10^{-6}$ is the threshold typically used by NASA to rule out an impact. e.g. http: //www . jpl . nasa.gov/asteroidwatch/newsfeatures.cfm?release=2013-017
} 
diameter close approachers but perhaps this is not too worrisome since they are unlikely to make it through Earth's atmosphere and cause serious ground damage. On the other hand, the opportunity for scientific study of more $2008 \mathrm{TC}_{3}$-like events (Jenniskens et al. 2009) is tremendous if the false alarm rate is small enough so, once again, follow-up observations are required for all these objects.

We estimated the false impactor rate (fig.7, right panel) with the single-survey system using the (Brown et al. 2013) PHO size frequency distribution appropriate for objects in this size range, our calculated close approacher survey efficiency (fig. 7, left panel), and the fraction of them that retain a non-zero impact probability on the (false) impact date (fig. 11). We find that the single all-sky survey system will generate 100s of false impactors per year for objects of $\lesssim 100 \mathrm{~m}$ diameter. Thus, rapid astrometric followup with other optical and radar facilities is imperative to reduce the false impactor rate to zero.

\section{Conclusions}

We have performed a simulation of a single all-sky asteroid survey to study the time evolution of the calculated impact probability for both real and false impactors. We also studied the utility of using two observatories at different locations to perform the survey to take advantage of the parallactic displacement in the detections of the same object.

As expected, the impact probability for impactors typically increases monotonically with time after discovery and is larger at the time of discovery for small objects that are detected closer to Earth and with less time to impact. We found that the impact warning time, the time interval between when the impact probability reaches -2 on the Palermo

Scale and when the impact takes place, increases with diameter according to $t_{\text {warn }} \propto D^{1.3}$ and developed a simple mathematical argument that the exponent should be in the range 
$[1.0,1.5]$

Close approaching asteroids can almost always be unambiguously identified as non-impactors but a small percentage will have a non-zero impact probability even on the day of (false) impact. i.e. the simulated survey on its own is unable to eliminate the impact risk. The fraction of objects for which a persistent impact risk exists at the time of impact increases with decreasing diameter of the object because the small objects have smaller observational arc lengths and concomitantly less precise orbit elements. The combination of the PHO size-frequency distribution with the probability of detecting false impactors suggests that the single all-sky system alone will generate 100s of potential impactors that must be ruled out with other followup facilities.

The calculated impact probability can take surprisingly long to reach $\sim 100 \%$ with just the results from a single low-precision astrometric survey. The impact probability may reach $100 \%$ only a few days before impact even for $300 \mathrm{~m}$ diameter objects detected a month in advance and imaged nightly thereafter .

Our simulations suggest that a 2-site survey is unnecessary, at least in terms of the incremental benefit in improving the impact probability calculation. The parallax afforded by this scenario only improves the impact probability calculation for a small fraction of the smallest asteroids detected shortly before impact. The 2-site survey offers many different cadence options and some can provide more efficient impact probability calculations than others. The derived impact probability was $\sim 10 \times$ higher (i.e. better) on the discovery night using the 'full-shift' cadence compared to the other two cadences. This suggests that a real survey that implements the 2 -site scenario should carefully test different cadences to select one that maximizes the efficiency and accuracy of the impact probability on the discovery night. The effect of survey cadence on the impact probability calculation is negligible on successive nights. 


\section{Acknowledgements}

We thank J. Tonry and L. Denneau of the ATLAS survey for assistance in characterizing and designing a representative ATLAS survey. D. Farnocchia was supported for this research by an appointment to the NASA Postdoctoral Program at the Jet Propulsion Laboratory, California Institute of Technology, administered by Oak Ridge Associated Universities

through a contract with NASA. Peter Vereš's Pan-STARRS MOPS Postdoctoral Fellowship was sponsored by the NASA NEOO grant No. NNX12AR65G. 


\section{REFERENCES}

Alvarez, L. W., Alvarez, W., Asaro, F., and Michel, H. V. 1980, Science, 208, 1095

Borovička, J., Spurný, P., Brown, P., Wiegert, P., Kalenda, P., Clark, D., and Shrbený, L. 2013, Nature, 503, 235

Bottke, W. F., Jr., Cellino, A., Paolicchi, P., and Binzel, R. P. 2002, Asteroids III

Bottke, W. F., Jr., Nolan, M. C., Melosh, H. J., Vickery, A. M., and Greenberg, R. 1996, Icarus, 122,406

Bowell, E., Hapke, B., Domingue, D., Lumme, K., Peltoniemi, J., and Harris, A. 1988, Asteroids II, 399

Brown, P. G. et al. 2013, Nature, 503, 238

Chao, E. C. T., Shoemaker, E. M., and Madsen, B. M. 1960, Science, 132, 220

Chesley, S. R. 2005, in IAU Colloq. 197: Dynamics of Populations of Planetary Systems, ed. Z. Knežević and A. Milani, p. 255

Chesley, S. R., Chodas, P. W., Milani, A., Valsecchi, G. B., and Yeomans, D. K. 2002, Icarus, 159,423

Chesley, S. R. and Spahr, T. B. 2004, in Mitigation of Hazardous Comets and Asteroids, ed. M. J. S. Belton, T. H. Morgan, N. H. Samarasinha, \& D. K. Yeomans , p. 22

Denneau, L. et al. 2013, PASP, 125, 357

Desmars, J., Bancelin, D., Hestroffer, D., and Thuillot, W. 2013, A\&A, 554, A32

Farnocchia, D., Bernardi, F., and Valsecchi, G. B. 2012, Icarus, 219, 41 
Grav, T., Jedicke, R., Denneau, L., Chesley, S., Holman, M. J., and Spahr, T. B. 2011, PASP, 123, 423

Gronchi, G. F. 2005, Celestial Mechanics and Dynamical Astronomy, 93, 295

Hodapp, K. W. et al. 2004, Astronomische Nachrichten, 325, 636

Jedicke, R. 1996, AJ, 111, 970

Jenniskens, P. et al. 2009, Nature, 458, 485

Kaiser, N. et al. 2002, in Society of Photo-Optical Instrumentation Engineers (SPIE) Conference Series, Vol. 4836, Society of Photo-Optical Instrumentation Engineers (SPIE) Conference Series, ed. J. A. Tyson and S. Wolff, p. 154

Kessler, D. J. 1981, Icarus, 48, 39

Larson, S., Brownlee, J., Hergenrother, C., and Spahr, T. 1998, in Bulletin of the American Astronomical Society, Vol. 30, Bulletin of the American Astronomical Society, p. 1037

Mainzer, A. et al. 2011, ApJ, 743, 156

Milani, A., Chesley, S. R., Chodas, P. W., and Valsecchi, G. B. 2002, Asteroids III, 55

Milani, A., Chesley, S. R., Sansaturio, M. E., Tommei, G., and Valsecchi, G. B. 2005a, Icarus, 173,362

Milani, A., Chesley, S. R., and Valsecchi, G. B. 1999, A\&A, 346, L65

Milani, A. and Gronchi, G. F. 2010, Theory of Orbit Determination (Cambridge University Press) 
Milani, A., Gronchi, G. F., Farnocchia, D., Knežević, Z., Jedicke, R., Denneau, L., and Pierfederici, F. 2008, Icarus, 195, 474

Milani, A. and Knežević, Z. 2005, Celestial Mechanics and Dynamical Astronomy, 92, 1

Milani, A., Sansaturio, M. E., Tommei, G., Arratia, O., and Chesley, S. R. 2005b, A\&A, 431,729

Opik, E. J. 1952, Irish Astronomical Journal, 2, 95

Ostro, S. J., Hudson, R. S., Benner, L. A. M., Giorgini, J. D., Magri, C., Margot, J. L., and Nolan, M. C. 2002, Asteroids III, 151

Pravec, P. and Harris, A. W. 2007, Icarus, 190, 250

Rabinowitz, D. L. 1991, AJ, 101, 1518

Standish, E. M. 1998, Interoffice Memorandum, 312.F-98-048, 1

Tonry, J. L. 2011, PASP, 123, 58

Valsecchi, G. B., Milani, A., Gronchi, G. F., and Chesley, S. R. 2003, A\&A, 408, 1179

Vereš, P., Jedicke, R., Wainscoat, R., Granvik, M., Chesley, S., Abe, S., Denneau, L., and Grav, T. 2009, Icarus, 203, 472

Vereš, P., Jedicke, R., Denneau, L., Wainscoat, R., Holman, M. J., and Lin, H. 2012, PASP

Virtanen, J., Muinonen, K., and Bowell, E. 2001, Icarus, 154, 412

This manuscript was prepared with the AAS IATEX macros v5.2. 


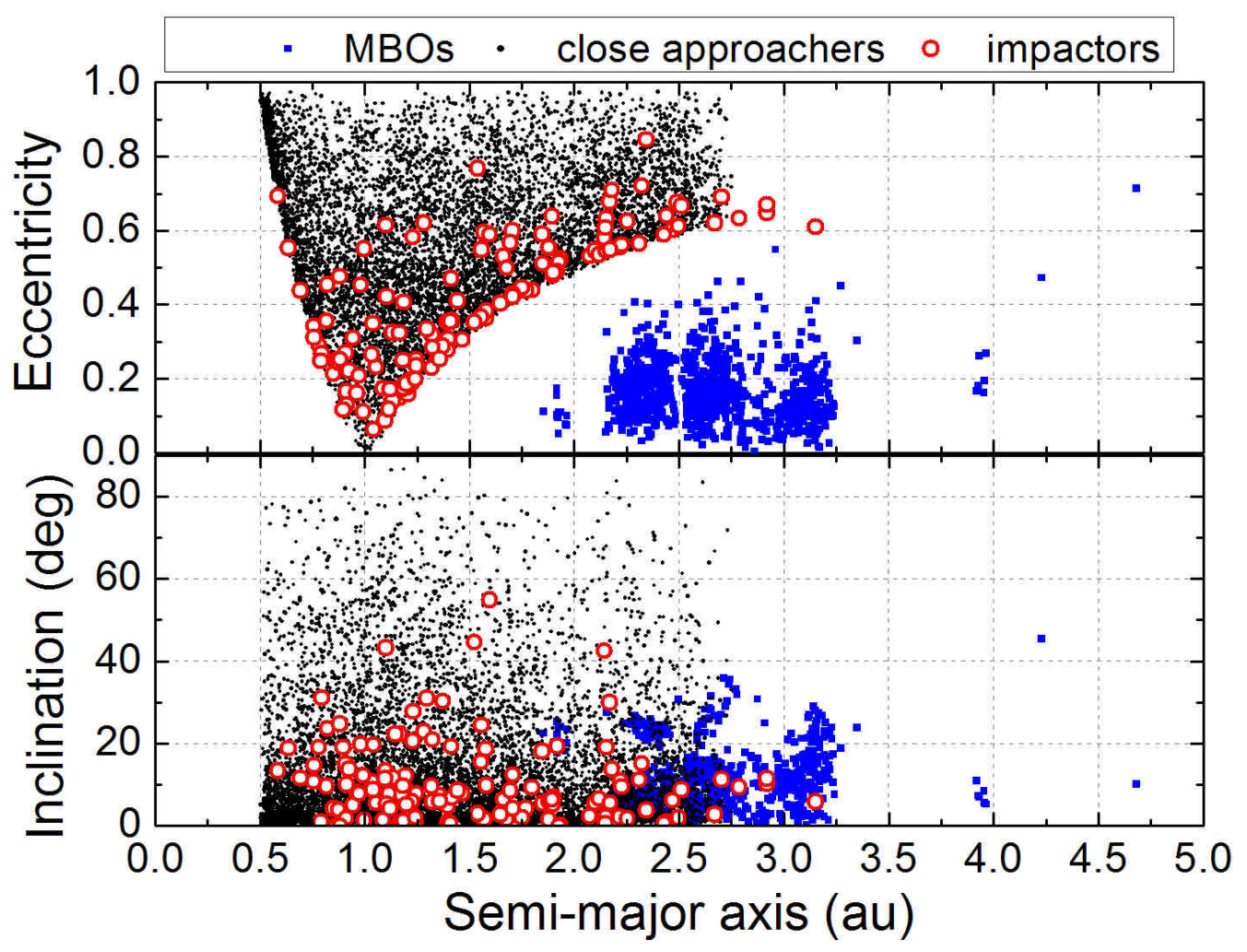

Fig. 1.- Eccentricity (top) and inclination (bottom) vs. semi-major axis for synthetic impactors (circles), close approachers (black dots) and main belt objects (grey squares). 


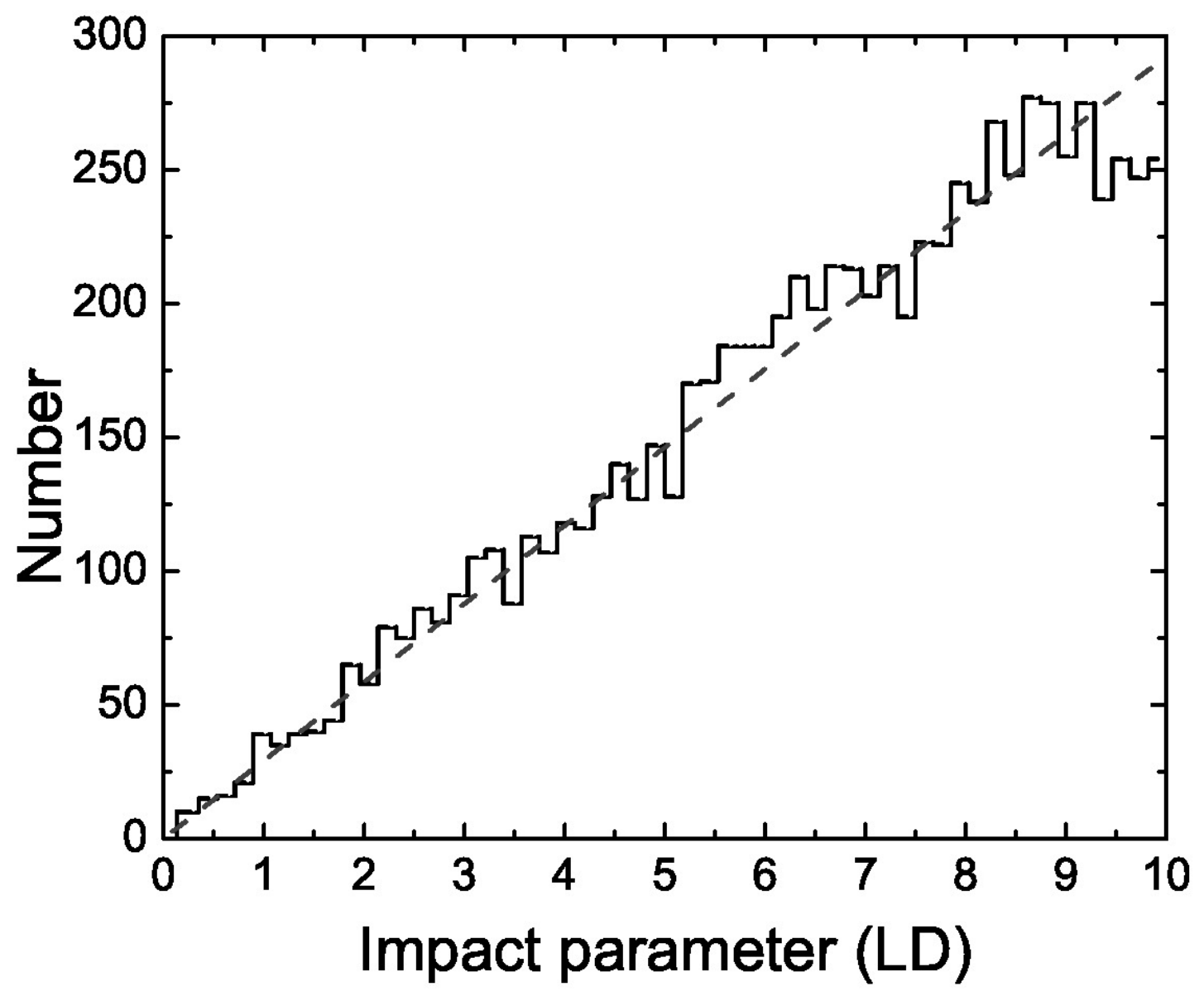

Fig. 2.- Impact parameters (closest approach distance to Earth) of the synthetic close approaching asteroids. The dashed straight line is a linear fit to the data. 

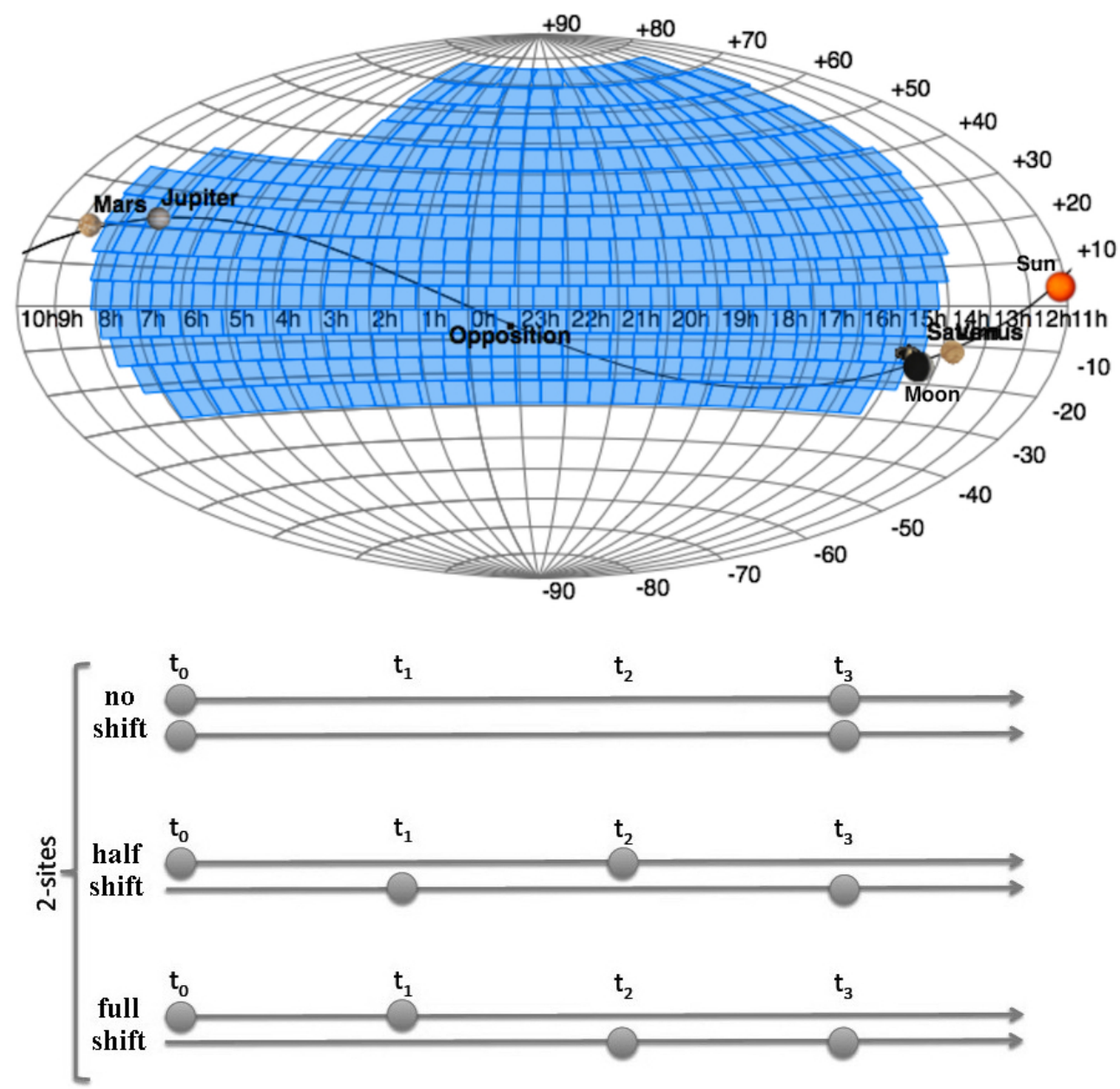

Fig. 3.- (top) One night of the synthetic ATLAS survey covering the entire night sky visible from Haleakala, Maui, Hawaii. Each shaded 'square' represents one bore site that is imaged 4 times/night. The dark solid line represents the ecliptic and the positions of some of the planets, Sun and Moon are represented with their images. The declination limit of $-30^{\circ}$ is roughly $40^{\circ}$ above the horizon as observed from Haleakala. (bottom) Time series for detections in tracklets for the single site 'quad' scenario and the 2 site 'no-shift', 'half-shift' and 'full-shift' scenarios. Each of the time steps represent a transient time interval (TTI). 


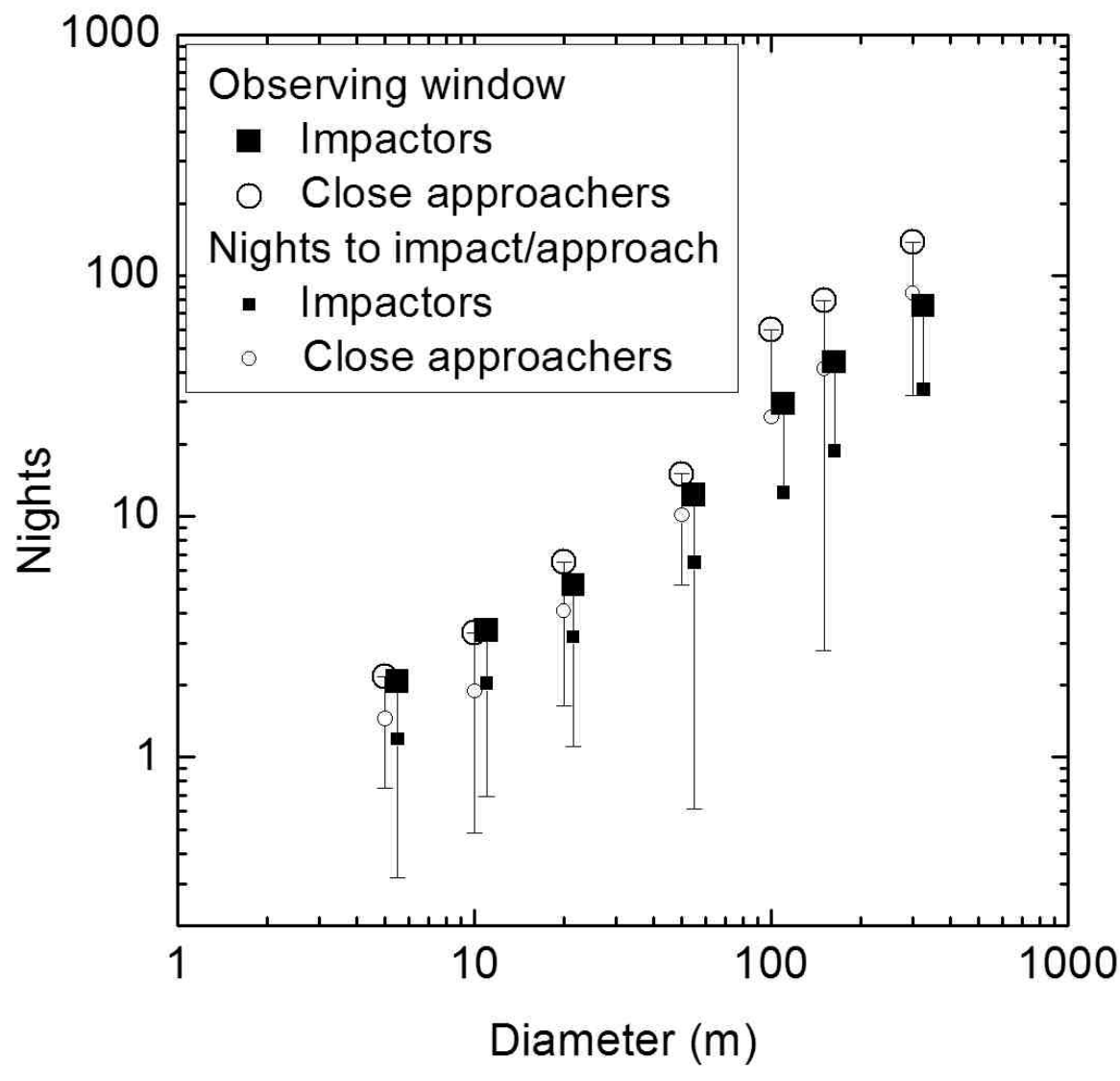

Fig. 4.- (large circles and squares) Observing window duration implemented in this study for close approachers and impactors on their final approach. (small circles and squares) Average number of nights to closest approach or impact. The upper limits of the error bars on these data points corresponds to the observing window durations by definition $(\$ 2.5)$. 

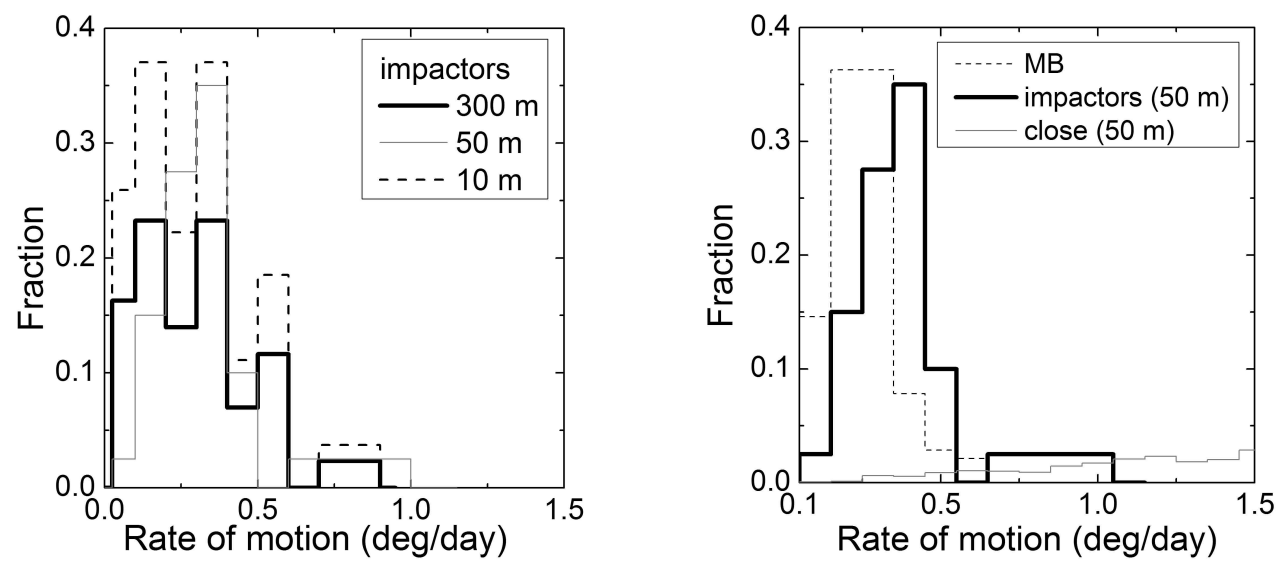

Fig. 5.- (left) Apparent rate of motion on the first night of observation for impactors with diameters of $10 \mathrm{~m}, 50 \mathrm{~m}$ and $300 \mathrm{~m}$. (right) Apparent rate of motion on the first night of observation for impacting, close approaching and main belt asteroids. The impactors and close approachers both have diameters of $50 \mathrm{~m}$ but the main belt asteroids have a realistic size-frequency distribution. 


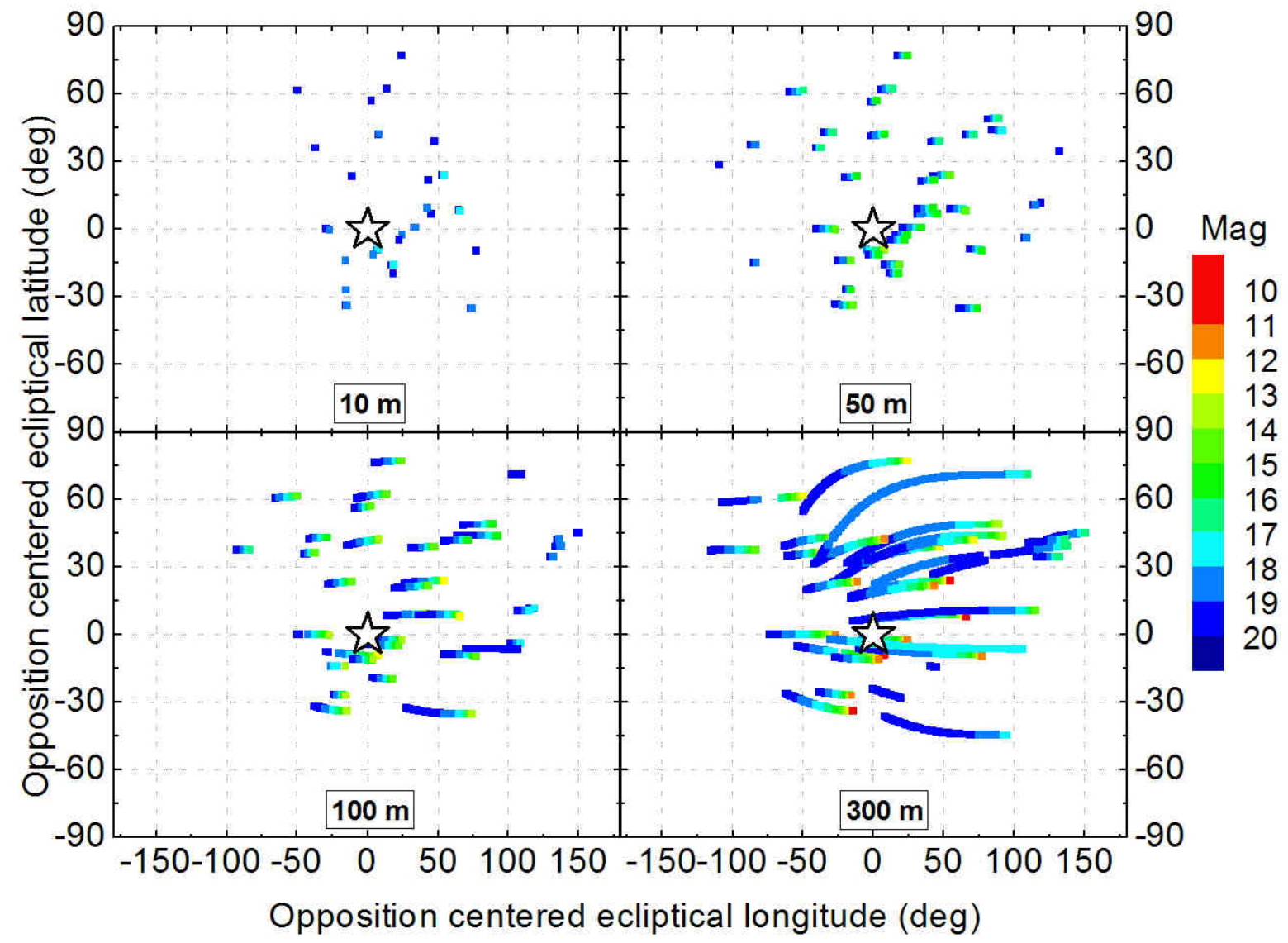

Fig. 6. - Time evolution of the sky plane position and apparent $V$ magnitude for all the detected synthetic impactors at 4 different sizes: $10 \mathrm{~m}, 50 \mathrm{~m}, 100 \mathrm{~m}$ and $300 \mathrm{~m}$. The coordinates are ecliptic opposition-centric with west to the right. The star symbol in the center represents opposition. 

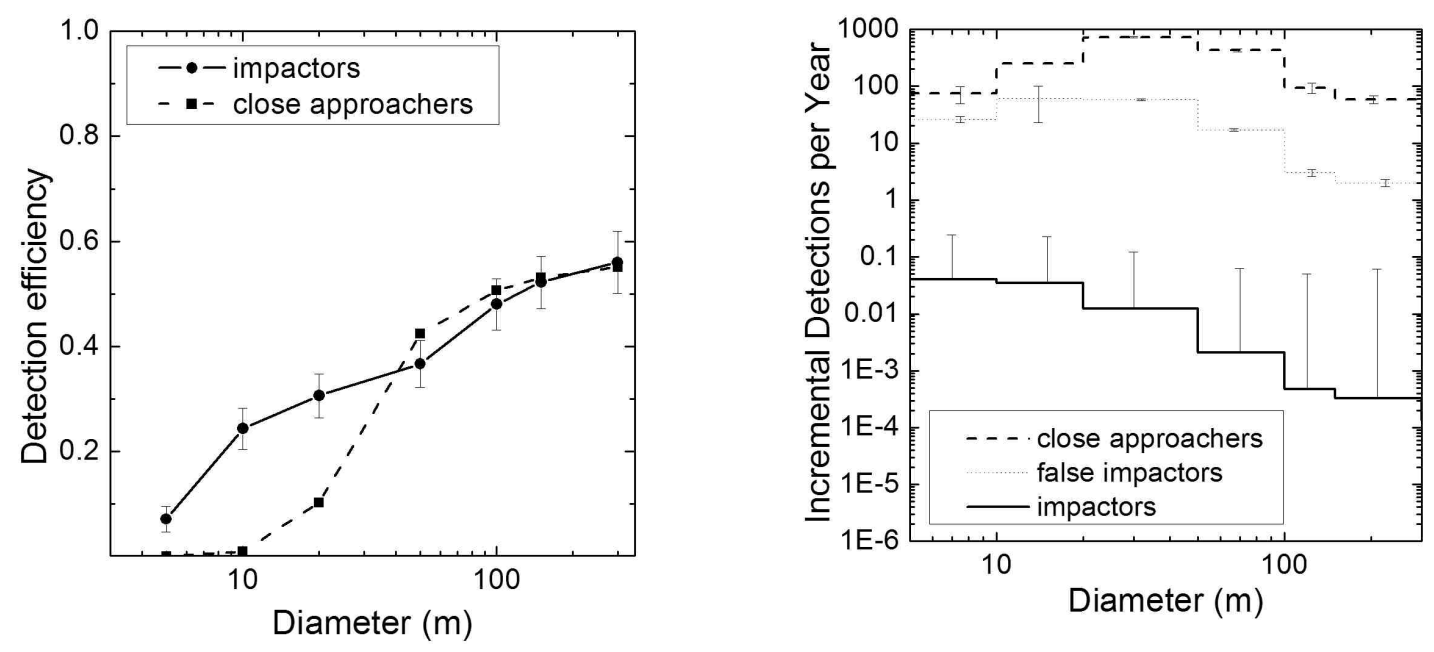

Fig. 7.- (left) Detection efficiency for synthetic impactors and close approachers as a function of object diameter and (right) our predicted incremental number of detections per year of impactors, close approachers, and false impactors (close approachers with a residual impact probability of $>10^{-6}$ on the day of closest approach — see fig. 11). Our calculation of the discovery rates used the Brown et al. (2013) impactor size-frequency distribution and impact rate. Note that the close approacher statistics only includes objects before closest approach for direct comparison to the impactors. 


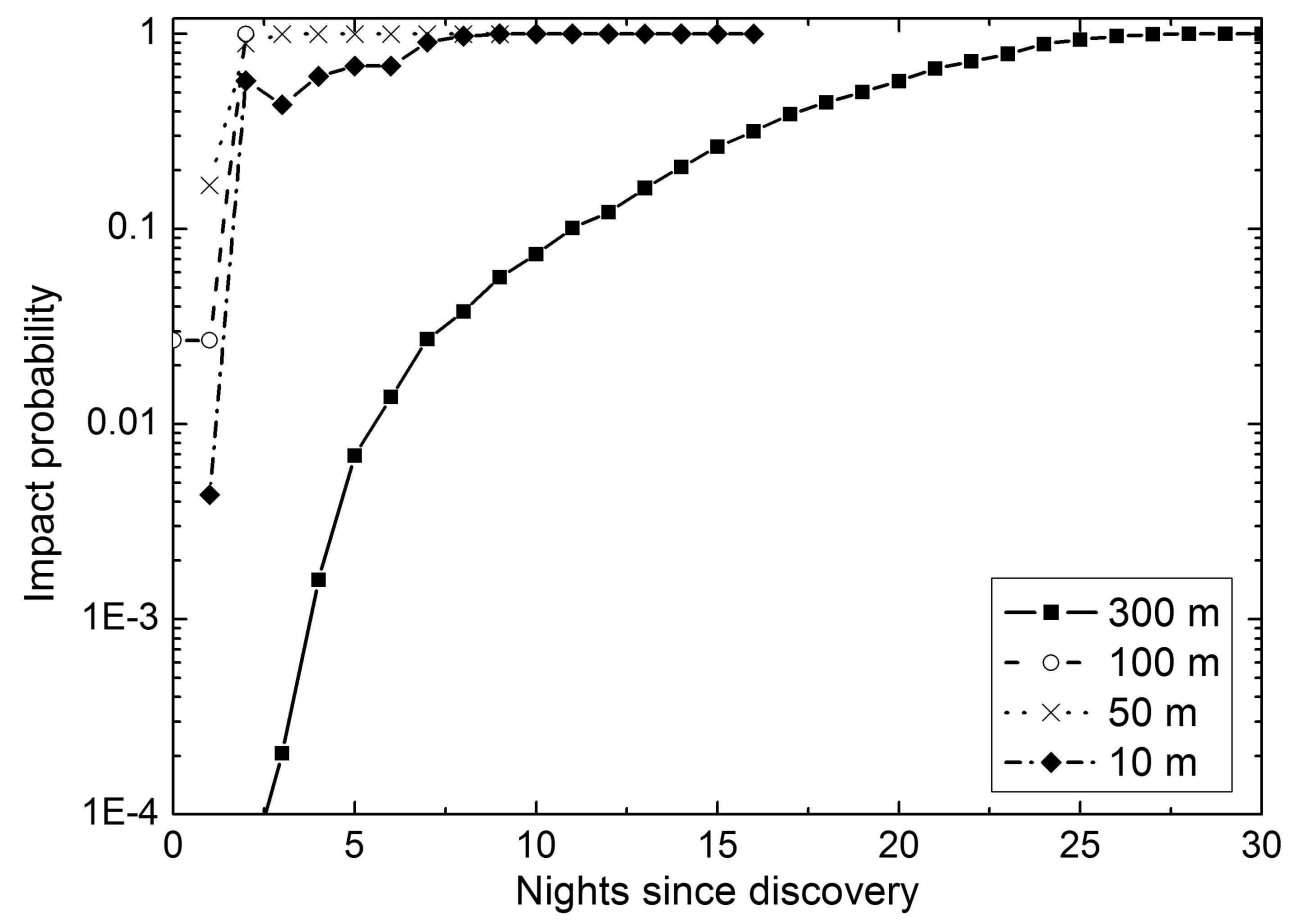

Fig. 8.- Impact probability time evolution for four synthetic objects of $10 \mathrm{~m}, 50 \mathrm{~m}, 100 \mathrm{~m}$ and $300 \mathrm{~m}$ diameter. 

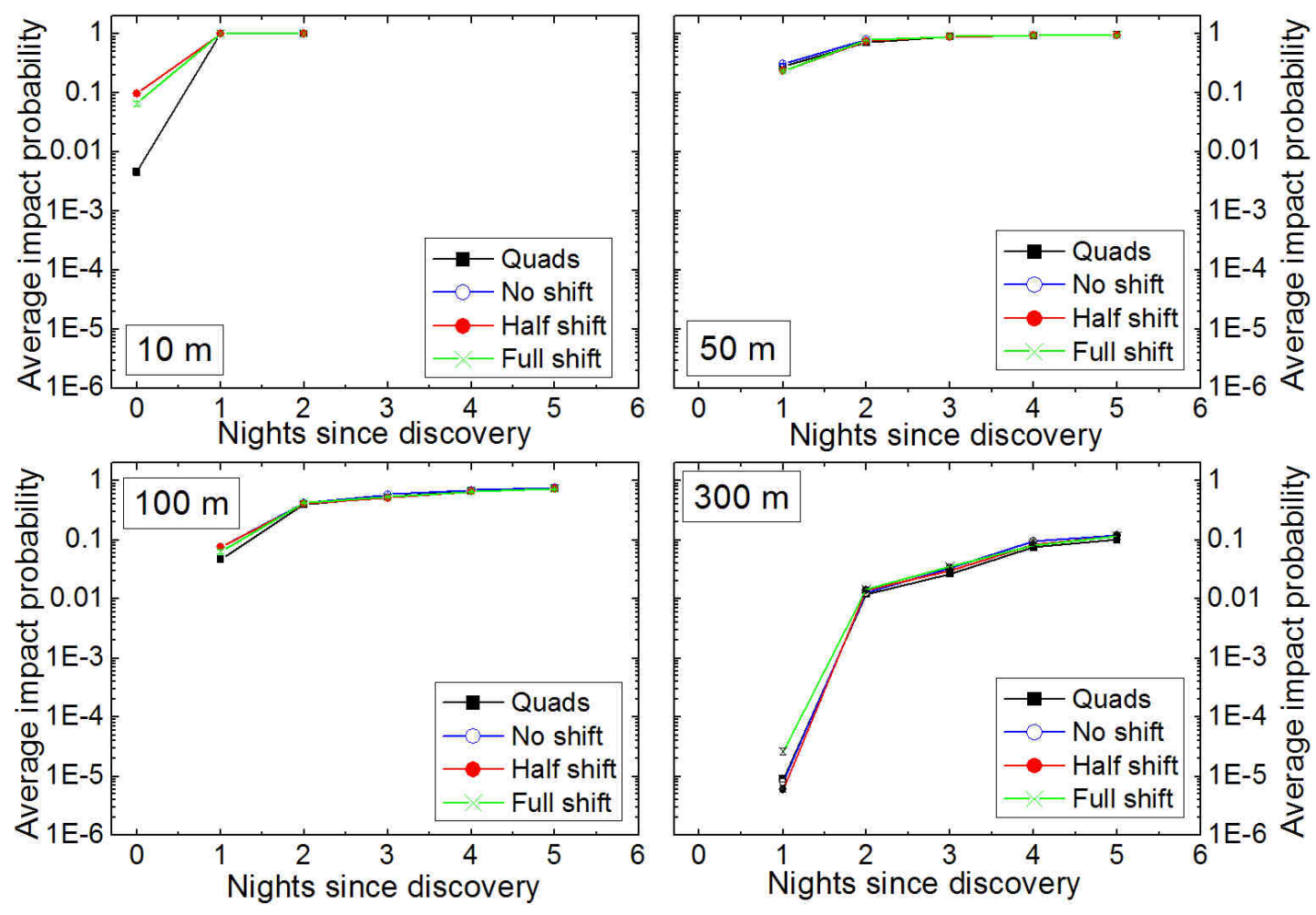

Fig. 9.- Average impact probability as a function of the number of nights since discovery for impactors of $10 \mathrm{~m}, 50 \mathrm{~m}, 100 \mathrm{~m}$ and $300 \mathrm{~m}$ diameter. The four curves correspond to the cadence scenarios illustrated in fig. 3 and discussed in $\$ 2.4$. The missing data points for night zero indicates that it was not possible to calculate the impact probability on the discovery night: this is not the same as claiming the objects have zero impact probability. 


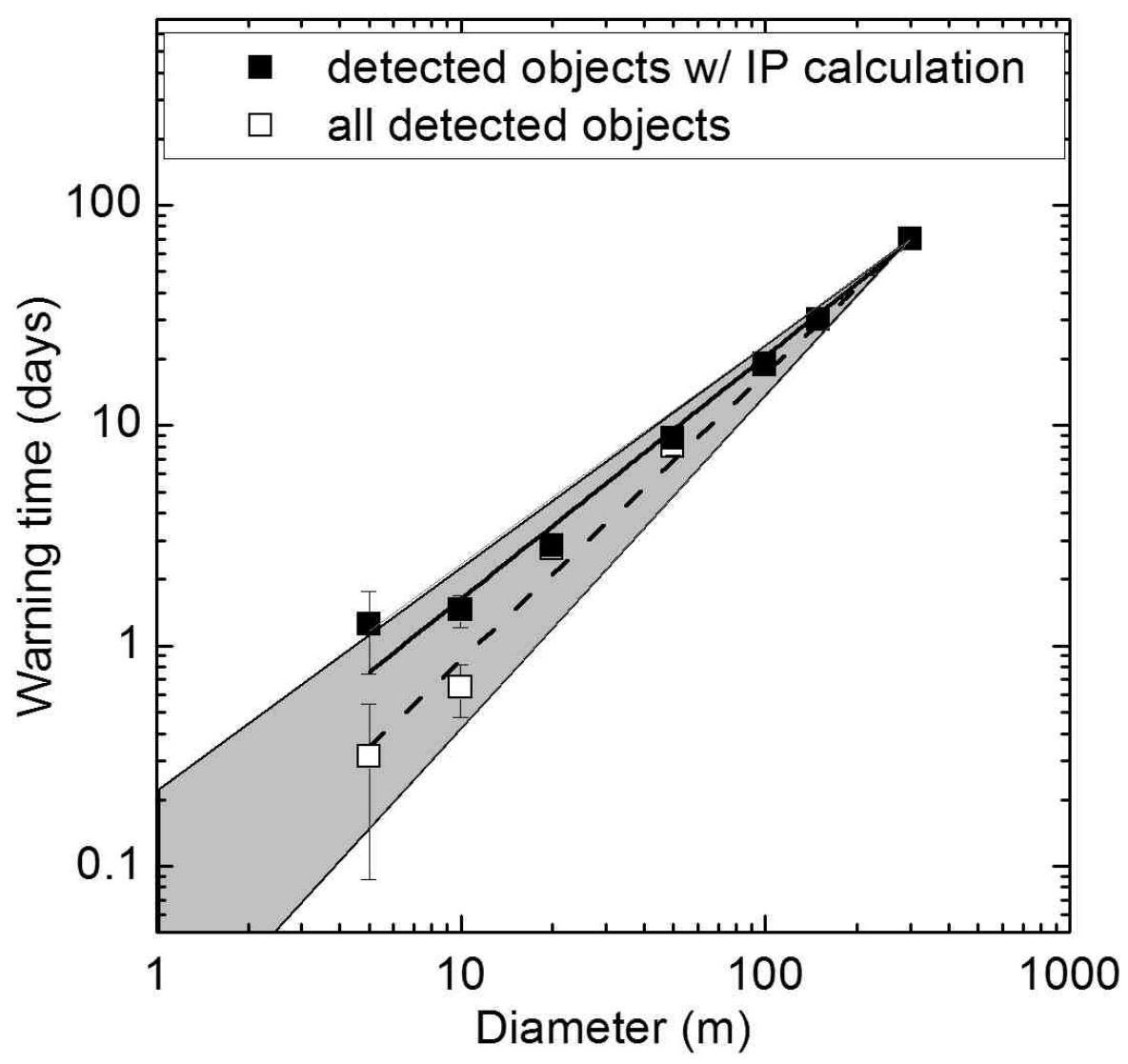

Fig. 10.- Impact warning time $t_{\text {warn }}$ as a function of impactor diameter $(D)$ for the ATLAS survey using the full-shift cadence (see fig. 3 and $\$ 2.4$ ). The error bars represent the standard error on the mean and are equal to or smaller than the data points for all but the two leftmost values. (dashed line) The fit to the data for all detected impactors (i.e. including those without calculated orbits and impact probabilities) with warning time given by $\log _{10}(t /$ days $)=(1.3 \pm 0.1) \log _{10}(D /$ meters $)-(1.4 \pm 0.2)$. (solid line $)$ The fit to the objects with calculated impact probability is $\log _{10}(t /$ days $)=(1.1 \pm 0.2) \log _{10}(D /$ meters $)-(1.0 \pm 0.4)$. The grey area represents the expected range with slopes in the range $[1.0,1.5]$ (see $\$ 3.3$ ) when anchored at $300 \mathrm{~m}$ diameter. 


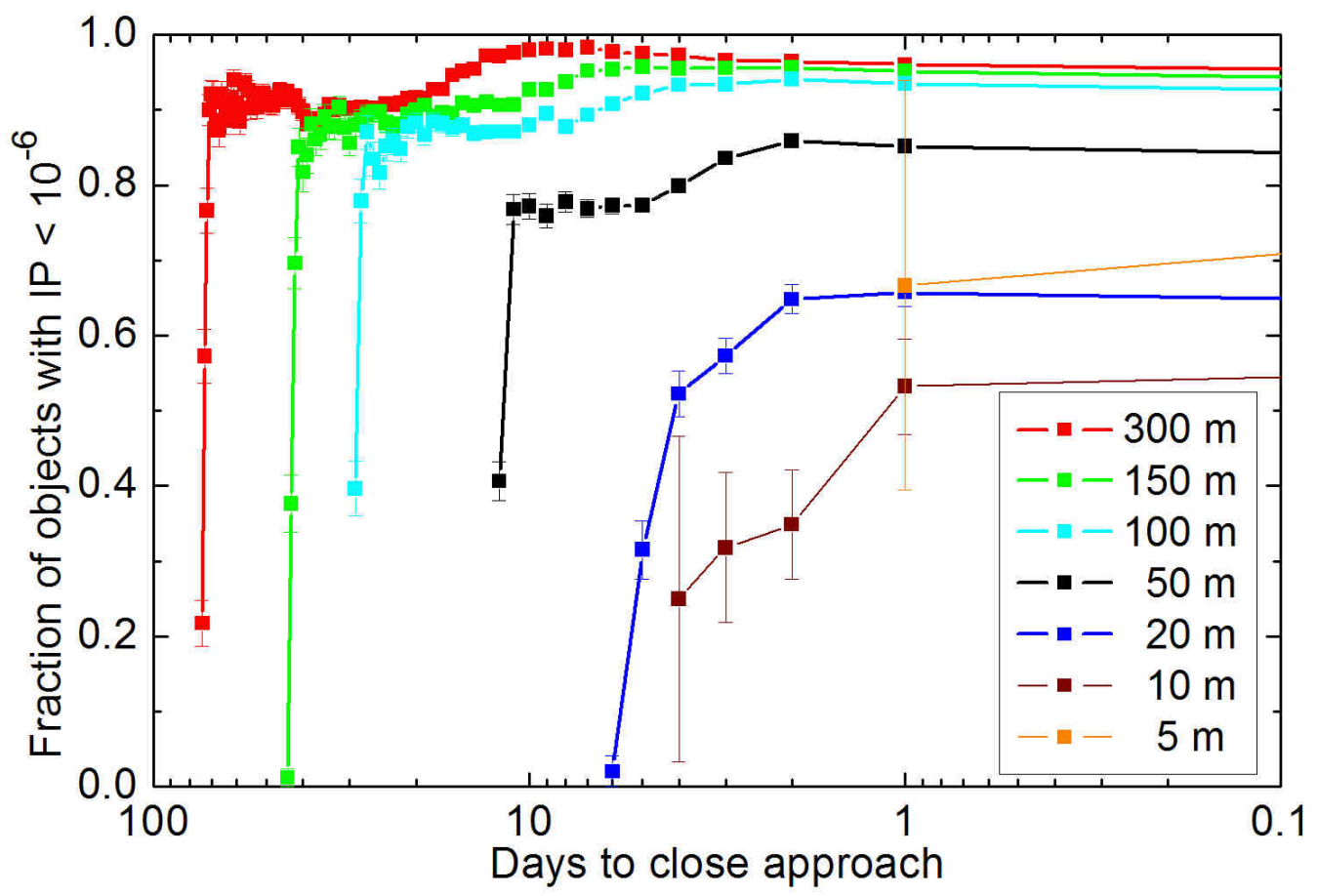

Fig. 11.- Fraction of close approaching asteroids for which an impact is ruled out (impact probability $<10^{-6}$ ) as a function of the number of days before close approach for four different asteroid diameters. The 'noisy' behavior on the left, corresponding to long times before impact, is mostly due to low number statistics. 